\title{
PORTABLE SOLAR SPECTRUM REFLECTOMETER FOR PLANAR AND PARABOLIC MIRRORS IN SOLAR THERMAL ENERGY PLANTS
}

\author{
Iñigo Salinas ${ }^{a *}$, Carlos Heras $^{\mathrm{a}}$, Carlos Alcañiz ${ }^{\mathrm{c}}$, David Izquierdo ${ }^{\mathrm{b}}$, Noelia \\ Martínez ${ }^{\mathrm{d}}$, Rafael Alonso ${ }^{\mathrm{a}}$ \\ ${ }^{a}$ Grupo de Tecnologías Fotónicas. Instituto de Investigación en Ingeniería de Aragón (I3A), Universidad de \\ Zaragoza, Zaragoza, Spain \\ ${ }^{b}$ Centro Universitario de la Defensa, Zaragoza, Spain \\ 'Abengoa Research, Soland A-472, Sanlúcar la Mayor, 41800, Sevilla, Spain \\ ${ }^{d}$ Abengoa Research, Paseo de la Castellana, Madrid, Spain
}

\begin{abstract}
This contribution presents a new field Solar Spectrum Reflectometer for solar-weighted specular reflectance characterization of planar, spherical or parabolic mirrors. This reflectometer is designed to provide fast and reliable field measurements and to be valid for any type of mirror currently installed in concentrated solar power systems, including parabolic trough, Stirling dish and central receiver power plants. The optical design of the Solar Spectrum Reflectometer, which includes 6 LEDs in the VIS-NIR band, is described, and its tolerance to variations in the geometrical parameters of the mirrors discussed and evaluated. The contribution of diffuse reflection and its impact on the measured reflectance is also calculated for different concentrated solar power systems.
\end{abstract}

\section{Keywords}

Portable solar reflectometer; solar plant surveying; specular reflectance; diffuse reflectance.

\section{Abbreviations}

STE: solar thermal energy

SSR: solar spectrum reflectometer

LED: light emitting diode

$\varphi_{\text {max }}$ : maximum aperture angle of the incident beam

$\alpha$ : angle of incidence on the glass

$\beta$ : angle of incidence on the mirror

$f:$ focal length

O: LED to lens distance

$\mathrm{O}$ : lens to detector distance

$\Phi$ : diameter

EP: entrance pupil

LE: entrance port

MO: mirror position

$\mathrm{h}$ : reflectometer to mirror relative displacement

$R$ : effective reflectance for a receiver

RSSR: effective reflectance for a reflectometer

$\mathrm{R}_{\text {spe }}$ : specular reflectance

$R_{\text {dif: }}$ diffuse reflectance captured by a receiver or a SSR

\footnotetext{
* Corresponding author.

E-mail address: isalinas@unizar.es
} 
$\Delta R$ : reflectance measurement error

I: intensity of a light source

$r$ : distance from mirror to a point on the receiver

D: shortest distance from mirror to receiver

$\mathrm{d}$ : width/diameter of the receiver

h: height of the location of a central receiver collector

I: height of a central receiver collector

\section{Introduction}

Solar thermal energy (STE) power plants concentrate the solar light in high temperature receivers for generation of electrical power or process heat. Different mirror designs to concentrate solar light are used: flat plane mirrors, parabolic dishes and parabolic curved troughs are the most common designs.

The performance of these mirrors is dependent on their solar-weighted reflectance and the specularity of the mirror surface. Reflectance in STE is defined as the ratio of incident optical power reflected by the mirrors into the acceptance half angle of the solar plant receiver, and is usually affected by dirt accumulation and micro surface imperfections.

Thus, frequent measurement of the reflectance of the mirrors used in solar concentrators is needed in order to determine the effect of accumulated dust or to detect any permanent degradation of their surface. Solar-weighted reflectance, which is the reflectance within a specified incidence and half-cone angle beam weighted by the solar spectrum, is a key parameter to evaluate the quality and performance of solar mirrors (Levinson et al., 2010; Meyen et al., 2009, 2010; Sutter, 2016). Accordingly, field solar-weighted reflectance measurement of mirrors becomes of great interest as it has a direct impact on the assessment and prediction of STE plant global performance and efficiency. Accurate determination of this parameter is required in the most advanced daily maintenance protocols as a sensor of the central status (Levinson et al., 2010; Meyen et al., 2010, SolarPaces Round Robin).

Commercially available equipment intended for this purpose, described in general as Solar Spectrum Reflectometers (SSR), often provide incorrect field solar-weighted specular reflectance measurements, as they can only measure reflectance at certain design conditions. When the thickness or the curvature of the mirror does not match the specifications used for the optical design of the instrument, its low tolerance to the value of the optical system entrance angle generates non-negligible errors (Polato and Masetti, 1988).

Depending on the optical design strategies, we can classify SSR in two main categories: hemispherical SSR and specular SSR. The former are equipped with an integrating sphere and are usually very reliable in laboratory scenarios when the correct reference standard is used (Fend et al., 2003; Montecchi, 2013; Roos, 1993); however, they require different configurations, including moving parts of the sphere for indirect acquisition of specular reflectance and large entrance ports to reduce the sensitivity of the instrument to mirror thickness (Polato and Masetti, 1988). In portable models, the adjustments necessary to measure mirrors with varied thicknesses and curvatures cannot be easily performed, so they are not usually suitable for plant surveying evaluations, which involve a large number of measurements that should be performed in a quick and simple way.

Specular SSR measure specular reflected light directly using an optical detector. Diffuse light is mainly discarded as its contribution is usually negligible, but obviously they show very low tolerance to changes in mirror design. Alignment and adjustment accessories can provide this 
tolerance (Sutter, 2016), but not without negatively impacting the SSR suitability for measurements performed in the field.

Usually, specular SSR are optically designed for planar mirrors in any soiling condition, but they underestimate specular reflectance on curved mirrors. On the other hand, portable hemispherical SSR achieve accurate specular reflectance values on any clean surface, but may overestimate specular reflectance when dirt accumulation is important, due to diffuse reflection not being discounted properly (Pettit and Freese, 1980).

This work proposes the use of collecting lenses in the detection system as an improvement on the specular SSR strategy. These lenses provide the necessary tolerance to changes in the geometrical dimensions of the mirrors, while helping to limit the impact of diffuse light on the measurement. This impact, though reduced, is not always negligible, so it will be necessary to establish a trade-off between the precision of the measurement and the adaptation of the reflectometer to fast and simple operation on the field.

According to these principles, we present a new optical design for a SSR, suitable for any planar, spherical or parabolic mirror currently installed in concentrated solar power thermo solar plants (Zhang et al., 2013), and which does not require moving parts or additional accessories. The advantages and limitations of this approach will be analyzed both theoretically and experimentally.

This design has been put to test in a portable device meeting all the requirements for plant surveying previously described, and commercialized as Condor (patent number WO2012010724).

The article is organized as follows. Section 2 describes the optical design of the instrument, with emphasis on the system aperture because of its impact on the final optical system tolerance. Section 3 includes theoretical and experimental studies of the tolerance of the device to variations in some geometrical parameters of the mirrors, as thickness and curvature radius. Section 4 explains the model used to evaluate the effect of the diffuse light in the reflectance measurements for different types of reflectometers and collectors. Section 5 uses that model to calculate the measurement errors due to diffuse light for the cases under study. Finally, conclusions are presented in section 6 .

\section{Optical design}

A suitable optical design is key for a field SSR instrument expected to work in any type of solar plant and measurement condition. In the new optical measurement device here described, the spectral reflectance is measured using 6 optical channels at different wavelengths, spanning from visible to near infrared, placed in line (Figure 1(a)). Each channel includes a light emitting diode (LED) as the light source, a reference detector, a lens and a signal detector as shown in figure $1(b)$.

The main function of the lens is to provide the required tolerance to changes in the mirror geometrical parameters. As will be discussed in section 3 , this tolerance is determined by the dimension of the measurement light beam diameter on the lens relative to the diameter of that lens (tolerance will be higher the smaller the beam is compared to the lens). To achieve a good ratio between these two parameters, the aperture of the beam is limited to a $\varphi_{\max }$ of $39 \mathrm{mrad}$ by using a fixed stop placed after the LED. This aperture determines the illuminated area on the lens surface and therefore the tolerance of the system. 

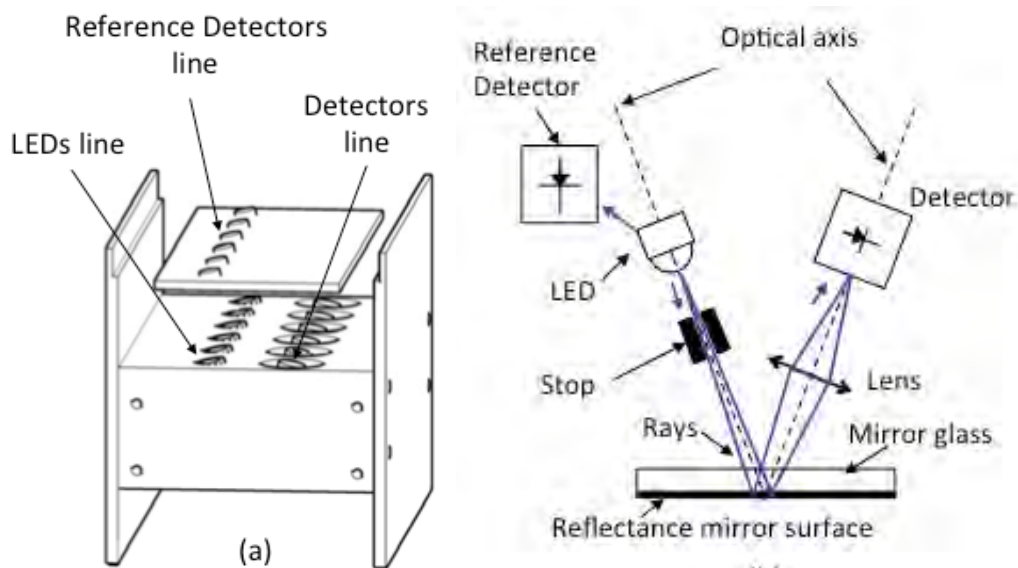

(b)

Figure 1. Basic Schematic set up: (a) device design, (b) single channel design.

The angle of incidence of the optical beam on the glass surface is $\alpha=12^{\circ}$, so, according to Snell's law, the angle of incidence on the mirror reflective surface is approximately $\beta=8^{\circ}$. Specularly reflected light is collected by a $12.5-\mathrm{mm}$ diameter, $15-\mathrm{mm}$ focal length $(\mathrm{f})$ fused silica biconvex lens. As will be discussed in section 4, this lens also collects a fraction of the diffusely reflected light, so its impact on the measured reflectance coefficient will be analyzed.

The LED is positioned at a distance $O$ from the lens so that the beam diameter on its surface, $\phi_{\text {beam_lens }}=2 O \cdot \tan \varphi_{\max }$, is half its diameter. It focuses the reflected light on a $9 \mathrm{~mm}^{2}$ effective area PIN photodiode detector (InGaAs or Si depending on the LED wavelength).

The paraxial optics diagram of one of the optical channels is shown in figure 2. In this figure, $f$ is the focal length of the lens, placed at the origin. The detector is located at the image point $O^{\prime}$ of the LED located at point $O$. The entrance pupil (EP) of the optical system is defined by the stop diameter and is located at a distance D1 from the lens. M0 indicates the position of the mirror in the design on ideal conditions ( $26.5 \mathrm{~mm}$ from the lens). The image of the detector is located at $O$ and works as the entrance port (LE), with a diameter given by the relationship:

$\phi_{L E}=\frac{O}{O^{\prime}} \phi_{d e t}$

with

$O=\frac{O^{\prime} \cdot f}{\left(O^{\prime}-f\right)}$

In order to compensate variations on the LED optical power, backwards emitted light from the LED is also detected as a reference signal, as shown in figure $1(\mathrm{~b})$. 


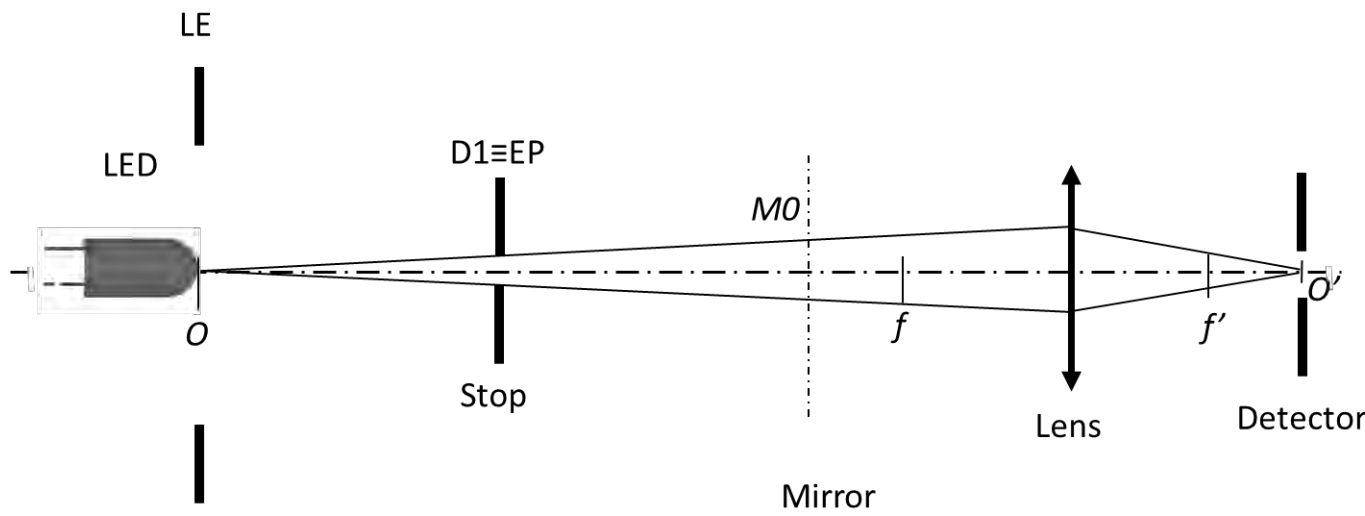

Figure 2. Single channel paraxial optics diagram for the design condition Mo. Origin is taken at the lens.

The number and wavelengths of the LED can be easily modified to span over different spectral ranges, depending on the accuracy required for the solar-weighted specular reflectance value. The example described here uses 6 optical channels at wavelengths of $405 \mathrm{~nm}, 535 \mathrm{~nm}, 650 \mathrm{~nm}$, $850 \mathrm{~nm}, 1100 \mathrm{~nm}$ and $1350 \mathrm{~nm}$.

The existence of various measurement wavelengths is an important advantage when the reflectometer is used to test samples with a solar reflectance either unknown or needing validation, for example prior to the installation of new mirrors in a solar plant. Even when testing for mirror soiling due to accumulation of dirt, where the loss of reflectivity is mainly due to scattering, a spectral measurement is recommended (Heller, 2013).

A plastic body encloses the optical system in order to block nearly all external incident light on the detectors. However, a small fraction of this light could still reach the detection system, limiting the measurement dynamic range depending on weather conditions and position relative to the sun. This limitation is avoided using a synchronous detection scheme: the optical sources are modulated at a known frequency and the detected signals are then processed to retrieve only the part of the signal corresponding to that frequency, thus eliminating noise and external light which won't be modulated.

A microcontroller is used to run the system, interact with the user through buttons and a LCD screen, and store the results of the measurements in its internal memory, resulting in a portable and autonomous device. The repeatability achieved with this kind of device is under $0.2 \%$ for measurements of solar-weighted reflectance.

\section{Tolerance to changes in geometry}

The optical design described above allows reflectance measurements with tolerance to changes both in thickness and curvature radius of mirrors. A measurement will be corrected assuring all rays forming the incident optical beam arrive at the detector, so this will be the condition defining the tolerance limits.

Figure $3(\mathrm{a})$ and figure $3(\mathrm{~b})$ show the ray tracing for two different conditions: $\mathrm{MO}$, in which the glass mirror thickness (a) and curvature radius (b) match those expected in the optical system design, and M1, in which glass mirror thickness (a) or curvature radius (b) are different.

As shown in figure 3(a), as the glass mirror thickness increases in $h$, the object optical distance varies in $\Delta X=2 X^{\prime}=2 h / \cos (\beta)$ and shifts in the vertical axis by 
$\Delta Y=Y^{\prime}=2 h \cdot \tan (\beta) \sin (\alpha)$. In a similar manner, in Figure (b), the mirror curvature 'displaces' the reflectometer a distance $h$ away from the surface plane on which the light is reflected, so the optical distance of the object varies in $\Delta X=2 X^{\prime \prime}=2 h / \cos (\alpha)$ and $\Delta Y=Y^{\prime \prime}=2 h \cdot \tan (\alpha) \sin (\alpha)$.

Both situations of glass thickness or curvature radius variation result in an increase $h$ in the distance between the device and the reflecting surface, which can be represented in the paraxial optics diagram as a shift in the position of the object from $\mathrm{O}$ to $\mathrm{O} 1$ given by $(\Delta \mathrm{X}, \Delta \mathrm{Y})$ with $\Delta X=2 h / \cos (\gamma)$ and $\Delta Y=2 h \cdot \tan (\gamma) \sin (\alpha)$, where $\gamma=\beta$ in the case of change in thickness and $\gamma=\alpha$ in the case of radius variation (figure 4).

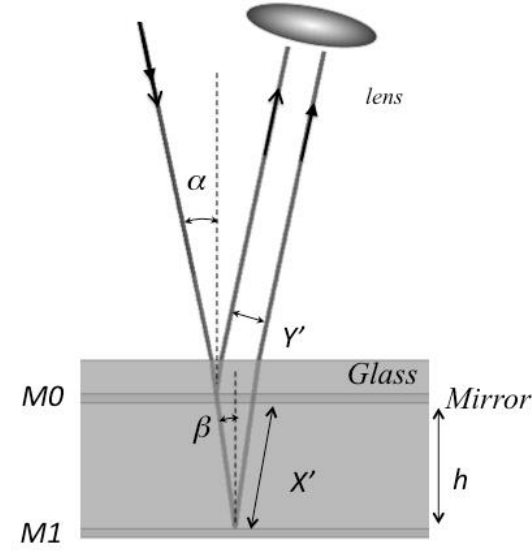

(a)

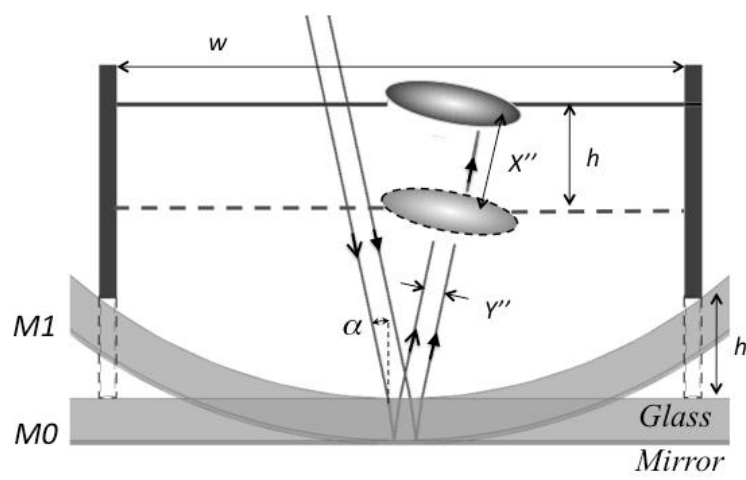

(b)

Figure 3. Ray tracing in design (M0) and out of design (M1) conditions

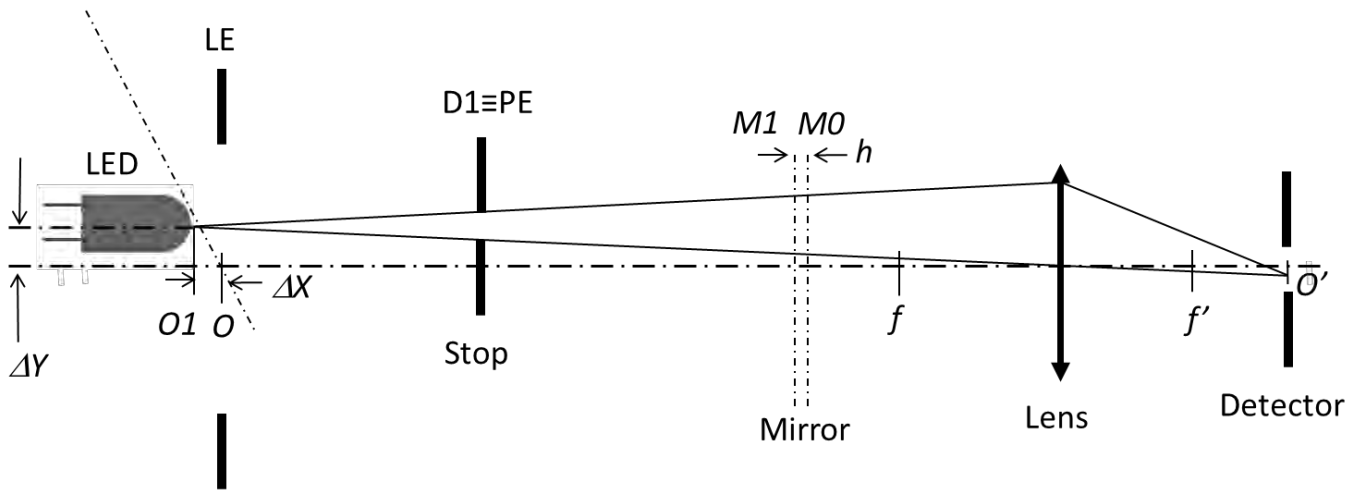

Figure 4. Single channel paraxial optics diagram for condition M1. Origin is taken at the lens.

As shown in figure 4, the tolerance of the system will be determined by the diameter and position of the beam spot on the lens, since the entrance port LE has a diameter and position that assures that all rays collected by the lens will reach the detector. As $h$ increases, the beam spot on the lens surface shifts vertically in $\Delta Y$ and also changes its diameter according to the following equation

$\phi_{\text {beam_lens }}=2(O+\Delta X) \tan \varphi_{\max }$ 
The circle-circle intersection area between that beam and the lens will be proportional to the optical power collected by the lens and hence define the tolerance of the instrument.

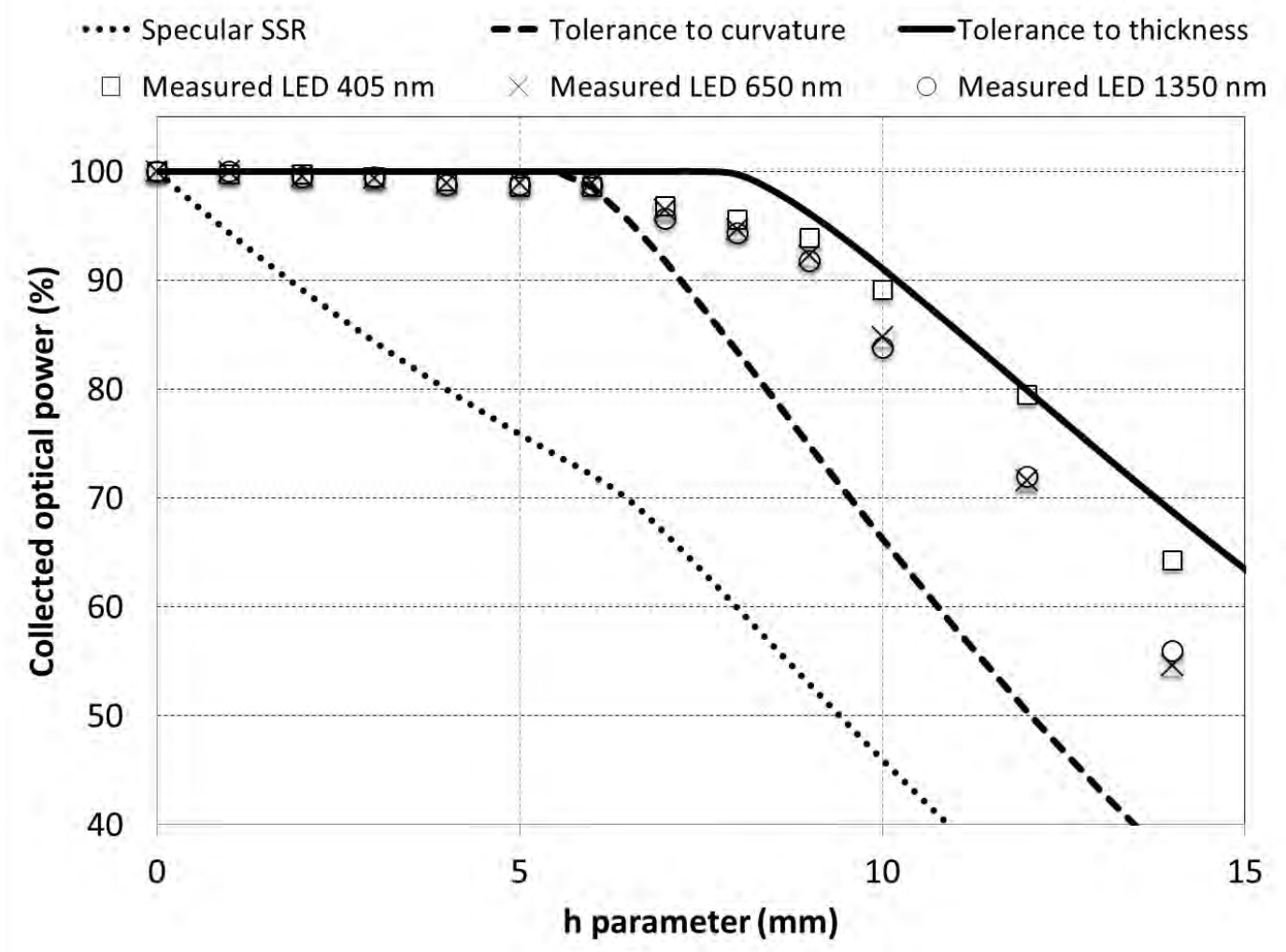

Figure 5. SSR calculated and measured tolerance to thickness and calculated tolerance to curvature.

In figure 5 we show the calculated optical power collected versus $h$ (tolerance to thickness) compared to measurements using different LEDs of our SSR (405 nm, $650 \mathrm{~nm}, 1350 \mathrm{~nm}$ ). The results report a tolerance to variations in $h$ (or mirror glass thickness) of $5 \mathrm{~mm}$ with deviations in the measured optical power under $1 \%$ and good matching with theoretical values. The maximum thickness of a solar mirror is $4 \mathrm{~mm}$, so this SSR is completely adequate to characterize the different types of solar mirrors in a reliable and fast way. Also, similar tolerance is achieved regarding a tilt of the device, due for example to dirt or wrong handling.

This data is also compared to the theoretical values for the tolerance to the curvature of the mirror (curvature radius $r$ and $h$ are geometrically related by $r=w^{2} / 8 h+h / 2$, with $w$ the body enclosure width. In our SSR, $w=120 \mathrm{~mm}$ ). Tolerance is ensured for curvature radius down to $r=300 \mathrm{~mm}(h=6 \mathrm{~mm})$ with $99.5 \%$ of the power collected, a value more than enough for solar mirrors characterization, where the minimum curvature radius is $3400 \mathrm{~mm}$ for parabolic through mirrors. For the sake of comparison, the tolerance of a purely specular SSR device, using direct detection without lens, with an aperture of $12.5 \mathrm{mrad}$, is also shown (Specular SSR).

\section{Diffuse reflection model}

The optical design described in the previous section provides tolerance to changes in the mirror geometry. However, the use of lenses to collect the reflected light implies that a fraction of the light produced by diffuse reflection will be captured along with the one belonging to specular reflection. This could result in overestimation of the reflectance coefficient, so it is convenient to evaluate this potential source of error. 
The following coefficients need to be defined for a proper discussion: $R$ (reflectance) is the ratio of incident light which is reflected by the mirror in the acceptance half angle of the receiver, and is the ideal value of the parameter to be determined. $R^{S S R}$ is the ratio of incident light that is reflected by the mirror in the acceptance half angle of the reflectometer, and it is the value measured by the device.

Both $R$ and $R^{S S R}$ are determined by two contributions: the specular and the diffuse reflection. So, we have $R=R_{\text {spe }}+R_{d i f}^{r e c}$ and $R^{S S R}=R_{\text {spe }}+R_{d i f}^{S S R}$. Where $R_{\text {spe }}$ is the specular power reflection coefficient of the mirror, and its contribution is the same in both cases since all of the light resulting from specular reflection is captured by the receiver and by the SSR. $R_{d i f}^{r e c}$ and $R_{d i f}^{S S R}$ are the fractions of incident light which are reflected diffusely by the mirror and captured respectively by the receiver and the SSR, and they can be different. The absolute error $\Delta R$ will be the difference between the measured reflectance $R^{S S R}$ and the real reflectance of the mirror $R$, and will vary with different soiling ratios:

$$
\Delta R=R^{S S R}-R
$$

To evaluate the contribution of diffuse reflection on $\Delta R$ we will consider a simple model for the light behavior on the reflective surface of the mirror: incident light can be absorbed by the mirror, reflected specularly or diffusely or attenuated by the soiling of the surface.

The portion of incident light absorbed directly by the mirror will be constant in time and related to the reflection coefficient measured for clean mirrors. The rest of the light will divide between specular reflection, diffuse reflection and attenuation due to dirt, with ratios depending on the mirror condition. In clean mirrors, specular reflection will be the only contribution, whereas dirt accumulation will decrease the amount of specularly reflected light, increasing diffuse reflection and attenuation.

To simplify, we will assume that both diffuse reflection and attenuation are proportional to the soiling ratio. We consider in our model that $85 \%$ of the light that is not specularly reflected will revert to diffuse light and $15 \%$ to attenuated light, as an average for the solar spectrum (Pettit and Freese, 1980). Other distributions can also be used depending on the soiling model considered.

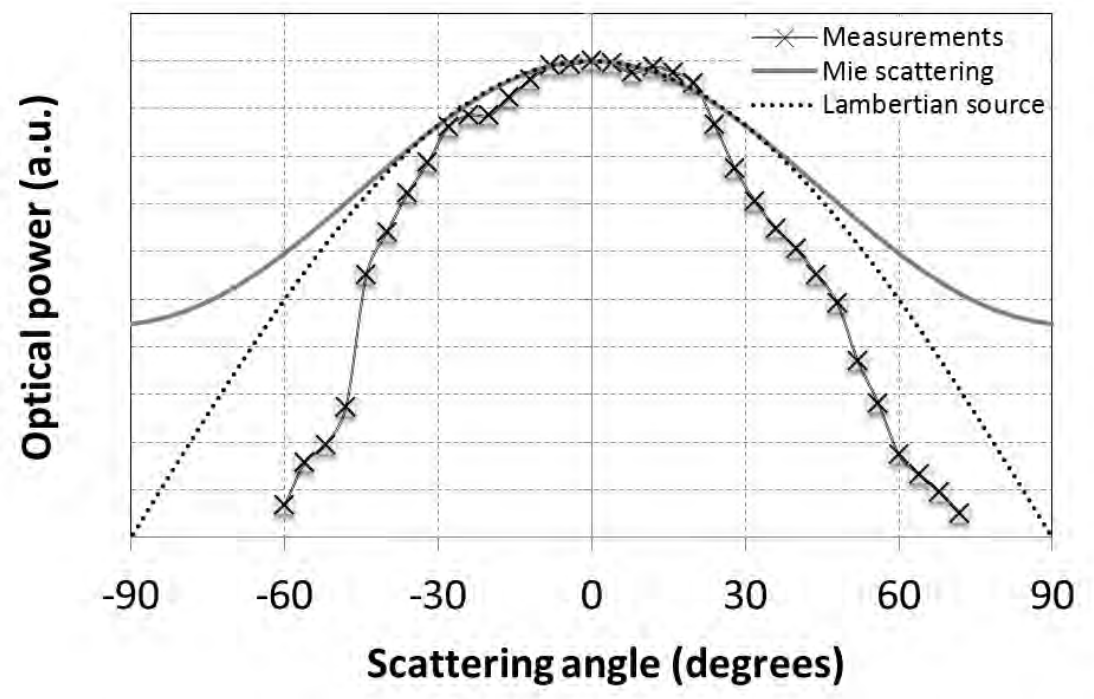

Figure 6. Scattering pattern for $100 \mathrm{~nm}$ particles at $650 \mathrm{~nm}$, compared to simulation and measurements of a Lambertian source at the same wavelength. 
Diffuse reflection is very difficult to model, as its angular distribution depends heavily on the size and shape of the scattering particles (Pettit, 1977). If the diameter of the particles is smaller than the wavelength of the incident light, Mie scattering for electromagnetic radiation can be approximated to Rayleigh scattering, and the diffuse reflection is similar to a Lambertian source of intensity $I(\theta)=I_{0} \cos \theta$ (figure 6). This case will be referred to as 'Lambertian' in the discussion below.

On the other hand, larger dirt particles would produce a more directional pattern. Figure shows that pattern for particles of 5 microns in average diameter, along with the diffuse reflection measured for a real dirt sample. Different soil compositions would mean changes on this scattering pattern, but the results of the simulations below should not vary radically for the most common cases. This scenery will be called 'Mie' from now on.

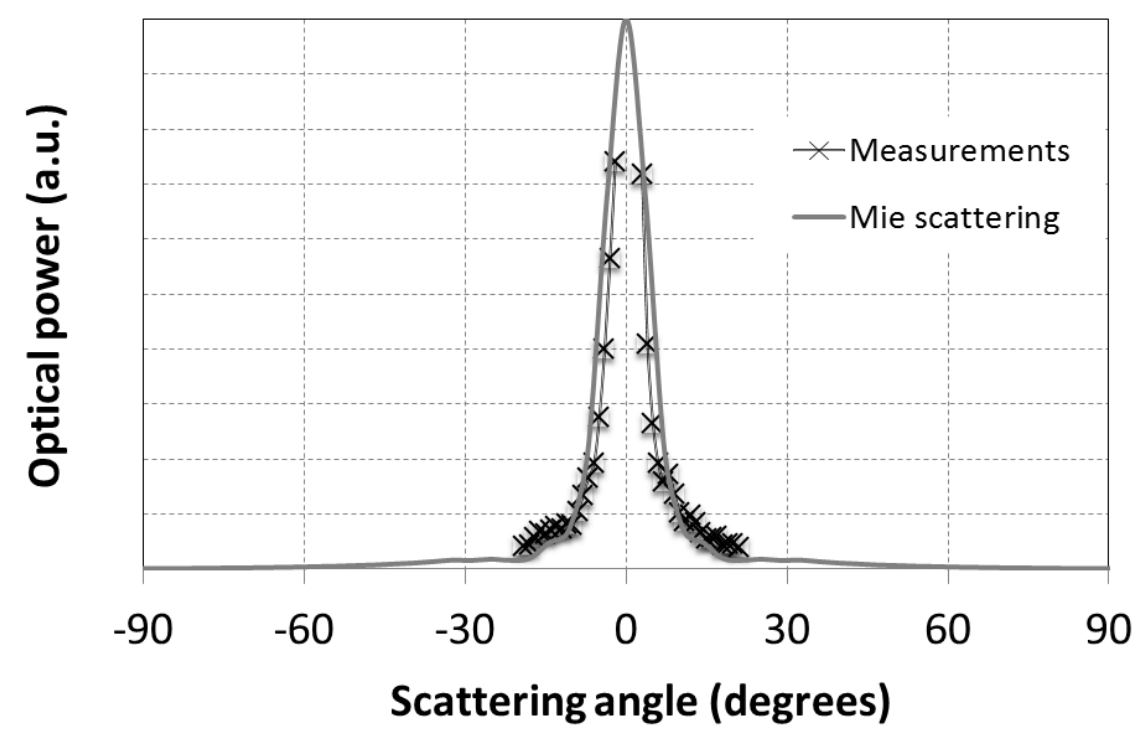

Figure 7. Mie scattering pattern for $5 \mu \mathrm{m}$ particles at $650 \mathrm{~nm}$, compared to measurements of a dirt sample at the same wavelength.

To calculate $R_{d i f}^{S S R}$ and $R_{d i f}^{r e c}$ we model the diffuse reflection as a source of intensity $I(\theta)=I_{0} f(\theta)$ , where $f(\theta)$ is either $\cos \theta$ ('Lambertian' scenery) or the function depicted in figure 7 ('Mie'). Then, the intensity of the total reflected diffuse light $I_{d i f}^{\text {tot }}$ is given, as shown in figure 8, by:

$I_{d i f}^{t o t}=\int_{0}^{\pi / 22 \pi} \int_{0} I(\theta) \sin \theta d \theta d \varphi= \begin{cases}\pi I_{0} & (\text { Lambertian }) \\ 0.070 I_{0} & (\text { Mie })\end{cases}$ 


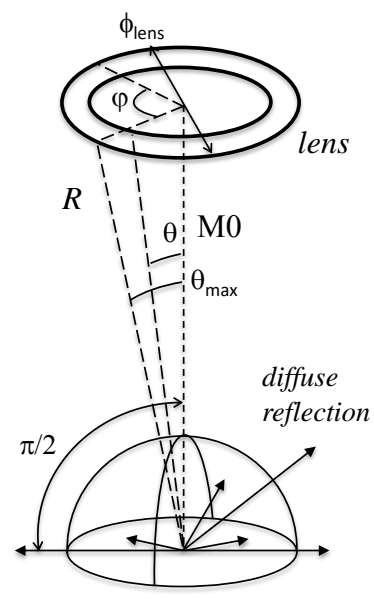

mirror

Figure 8. Diffuse reflection calculation for SSR and Stirling dish.

For the reflectometer, the acceptance half angle is determined by the lens diameter as

$$
\theta_{\max }=\arctan \left(\frac{\phi_{\text {lens }}}{2 M 0}\right)
$$

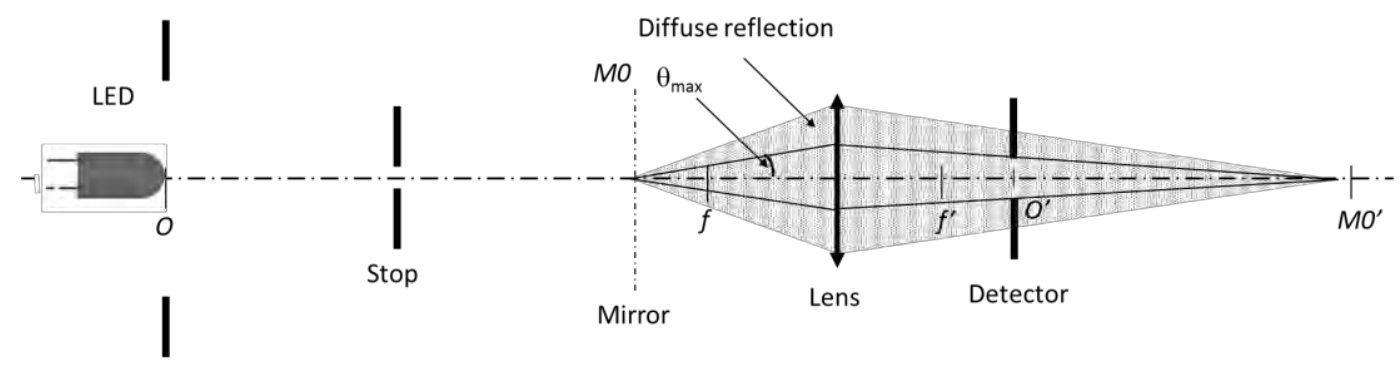

Figure 9. Diffuse reflection captured in the optical system of the SSR.

However, the diffuse reflection takes place at the mirror instead of at the optical system entrance, so not all of the incident rays on the lens will reach the detector. For points on the system axis, as the case depicted in Figure 9, the effective acceptance half angle will be reduced to $\theta_{\max }=145 \mathrm{mrad}$. Thus, the intensity of the collected diffuse light $I_{d i f}^{S S R}$ will be:

$I_{d i f}^{S S R}=\int_{0}^{\theta_{\max }} \int_{0}^{2 \pi} I(\theta) \sin \theta d \theta d \varphi=\left\{\begin{array}{l}0.066 I_{0}(\text { Lambertian }) \\ 0.022 I_{0}(\text { Mie })\end{array}\right.$

The ratio between $\mathrm{Eq}(5)$ and $\mathrm{Eq}(6)$ implies that, for a Lambertian source, $2.1 \%$ of the diffusely reflected light is collected by the reflectometer whereas it reaches 31\% for a 'Mie' scattering sample.

This model can be also used to estimate the performance of other types of reflectometer designs. A hemispherical reflectometer using integrating spheres will capture all the diffuse light, so $R_{d i f}^{S S R}=R_{d i f}$, which will result in important overestimations of $R$. For the specular design, $0.016 \%$ or $0.69 \%$ of the diffuse light will be captured, depending on the scattering model, using a typical value of acceptance half angle of $12.5 \mathrm{mrad}$ and $\mathrm{Eq}(6)$. 
At the same time, solar receivers using different configurations will capture different fractions of the diffusely reflected light, resulting in different values for $R_{d i f}^{r e c}$. In the case of parabolic trough collectors (PTC), the intensity of the diffuse light collected $I_{d i f}^{P T}$ can be evaluated from the differential solid angle subtended by a differential area of the receiver $d \Omega_{P T}$. According to Figure (a):

$d \Omega_{P T}=\frac{\hat{r} \cdot d \vec{S}}{r^{2}}=\frac{D}{r^{3}} d x d y$, with $r=\sqrt{x^{2}+y^{2}+D^{2}}$ and $\theta=\cos ^{-1}\left(\frac{D}{r}\right)$

We consider an infinite line of collectors, so $y$ is integrated from $-\infty$ to $+\infty$ :

$I_{d i f}^{P T}=\int I \mathrm{~d} \Omega_{P T}=I_{0} \int_{-d / 2}^{d / 2} \int_{-\infty}^{\infty} \frac{D}{r^{3}} f(\theta) d x d y=\left\{\begin{array}{l}0.065 I_{0} \text { (Lambertian) } \\ 0.007 I_{0}(\text { Mie })\end{array}\right.$

Considering standard receiver tube dimensions of $d=7 \mathrm{~cm}$ and $D=171 \mathrm{~cm}$, either $2 \%$ or $10 \%$ of the diffusely reflected light is collected by the receiver, depending on the scattering model.

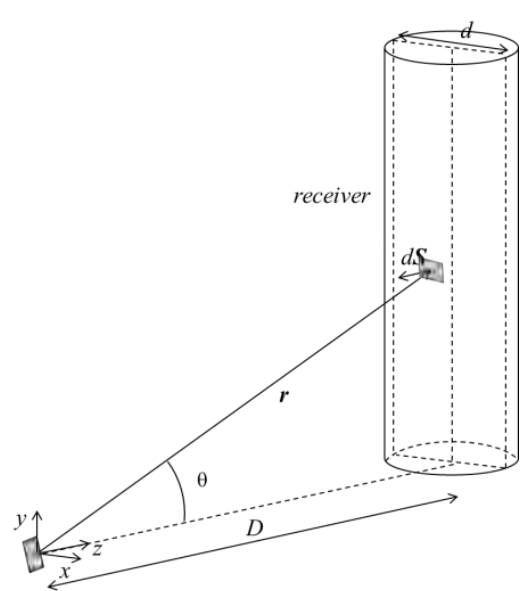

(a)

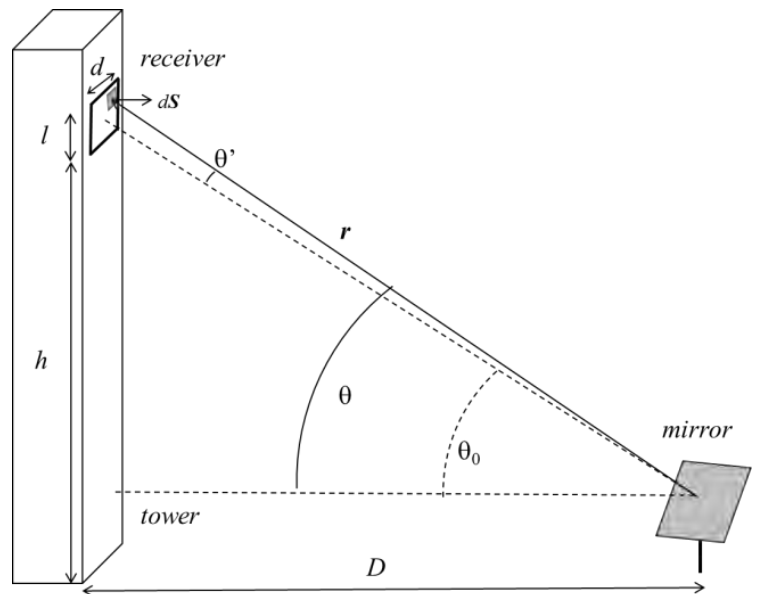

(b)

Figure 10. Diffuse reflection calculation for PTC (a) and central receiver plants (b).

In the case of central receiver plants, similar calculations can be performed using the geometrical analysis shown on 10(b):

$$
\begin{aligned}
& d \Omega_{\text {tow }}=\frac{D}{r^{3}} d x d y, \text { with } r=\sqrt{x^{2}+y^{2}+D^{2}} \text { and } \theta=\cos ^{-1}\left(\frac{D}{r}\right) \\
& y_{\text {max }}=h+l, y_{\text {min }}=h, x_{\max }=d / 2, x_{\min }=-d / 2 ; \theta^{\prime}=\theta-\theta_{0}, \text { with } \tan \theta_{0}=\frac{h+l / 2}{D} \\
& I_{\text {dif }}^{\text {tow }}=\int I \mathrm{~d} \Omega_{\text {tow }}=I_{0} \int_{-d / 2}^{d / 2} \int_{\mathrm{h}}^{h+l} \frac{D}{r^{3}} f(\theta) d x d y= \begin{cases}0.002 I_{0} & (\text { Lambertian }) \\
3.95 \cdot 10^{-4} I_{0} & (\text { Mie })\end{cases}
\end{aligned}
$$

which means that only between $0.06 \%$ and $0.57 \%$ of the diffusely reflected light is collected by the tower (considering $d=12 \mathrm{~m}, \mathrm{l}=10 \mathrm{~m}, \mathrm{D}=100 \mathrm{~m}$ and $\mathrm{h}=150 \mathrm{~m}$ ). For $\mathrm{D}=1000 \mathrm{~m}$, the percentages would be even lower, $0.003 \%$ and $0.14 \%$. 
The Stirling dish collector requires the same calculations than the SSR, substituting $\phi_{\text {lens }}$ in Figure by the diameter $d$ of the receiver and $d_{M}$ by the distance $D$ between the dish and the receptor.

Thus, $\theta_{\max }=\arctan \left(\frac{d}{2 D}\right)=20 \mathrm{mrad}(d=20 \mathrm{~cm}, D=5 \mathrm{~m})$ :

$I_{d i f}^{S t i}=\int_{0}^{\theta_{\max }} \int_{0}^{2 \pi} I(\theta) \sin \theta d \theta d \varphi=\left\{\begin{array}{l}1.3 \cdot 10^{-3} I_{0}(\text { Lambertian }) \\ 1.2 \cdot 10^{-3} I_{0}(\text { Mie })\end{array} \quad \mathrm{Eq}(9)\right.$

So either $0.04 \%$ or $1.7 \%$ of the diffuse reflection is collected by the dish.

\begin{tabular}{|l|l|l|}
\hline \multirow{2}{*}{ Optical system } & \multicolumn{2}{|l|}{ \% of diffuse reflection collected } \\
\cline { 2 - 3 } & Lambertian & Mie \\
\hline Specular SSR & 0.016 & 0.69 \\
\hline Lens SSR & 2.1 & 31 \\
\hline Hemispherical SSR & 100 & 100 \\
\hline Parabolic through collector & 2.0 & 10 \\
\hline Central receiver plant & $0.003-0.06$ & $0.14-0.56$ \\
\hline Stirling dish & 0.04 & 1.7 \\
\hline
\end{tabular}

Table 1. Performance of various SSR and collectors concerning diffuse reflection.

\section{Tolerance to diffuse reflection}

Using these values, summarized in table 1 , it is possible to calculate the error in the measurement of $R$ for different collectors and using different SSR designs.

Figure and Figure show calculated values for the measurement error $\Delta R=R^{S S R}-R$ as defined in Eq(4) versus the reflection coefficient $R=R_{s p e}+R_{d i f}^{r e c}$ of the mirror, which is directly related to the soiling ratio of the surface. We consider a standard value of $94 \%$ for the specular reflectance $R_{s p e}$ of the clean mirror, which will decrease as the soiling ratio and the diffuse reflection increase. The grey area in the charts indicates the minimum reflectance value usually admitted in STE plants $(R \approx 89 \%)$. Values lower than this demand mirror cleaning and are not usually found in the field in an operational plant.

For the three types of solar plant evaluated, the hemispherical reflectometer substantially overestimates the value of $R$. As it captures all of the diffuse light, the measurement obtained is almost independent of the soiling ratio, and decreases only due to the attenuation produced by the dirt. This results in errors around $7 \%$ for a real $R$ of $86 \%$ and $4 \%$ in operation conditions.

The specular reflectometer, on the other hand, discards almost all the diffusely reflected light, and so provides a better estimation of the real value of $R$. In the case of the PTC, however, the measurements obtained with a specular SSR slightly underestimate the effective value of $R$. This fact is more evident when using the 'Mie' scattering model, because the collector captures $10 \%$ of the diffuse reflection while the SSR only measures a $0.69 \%$. It is also important to remember that in this case the tolerance to geometrical changes in the mirrors will be nearly inexistent, as has been shown previously in figure 5 .

Using the design presented in this paper (Lens SSR), the measured reflectance coefficients are also overestimated, since the amount of diffuse light captured in the reflectometer is larger than in the collectors. Nevertheless, this overestimation is negligible if the diffuse reflection is 
Lambertian, as the error is under $0.2 \%$ even for dirt accumulation on mirrors resulting in effective reflectance coefficients $8 \%$ lower than the original values. If the 'Mie' scattering approximation is used the deviation is larger, but will always be less than $2 \%(<1.35 \%$ in operation conditions). The better results are obtained for the parabolic through collectors, as they also capture a sizeable portion of the diffuse light. For these collectors, the maximum error in operation is around $1 \%$.
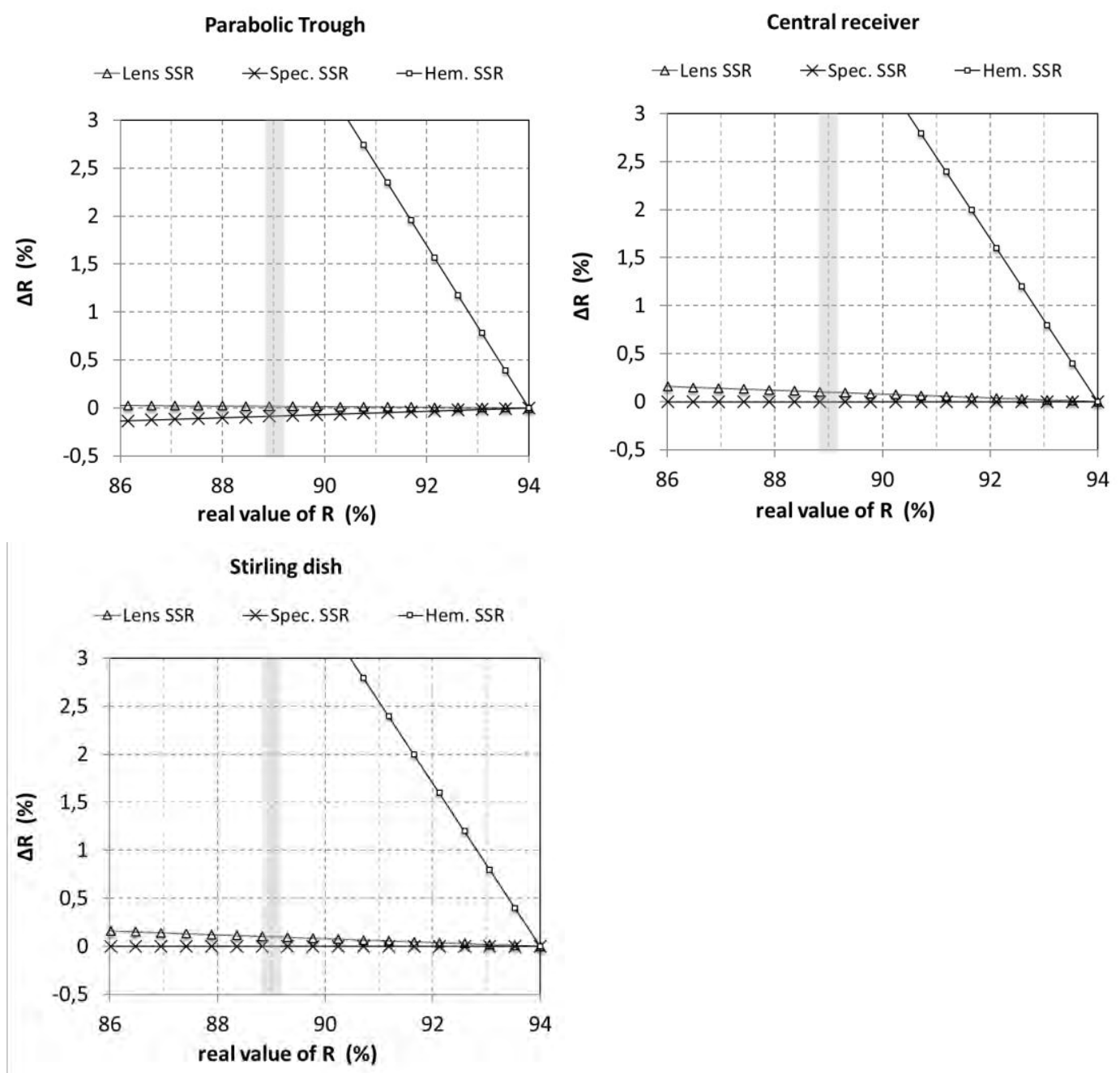

Figure 11. Error $\Delta R$ versus specular reflection coefficient for the different types of reflectometers and collectors, using a Lambertian model for the diffuse reflection. 

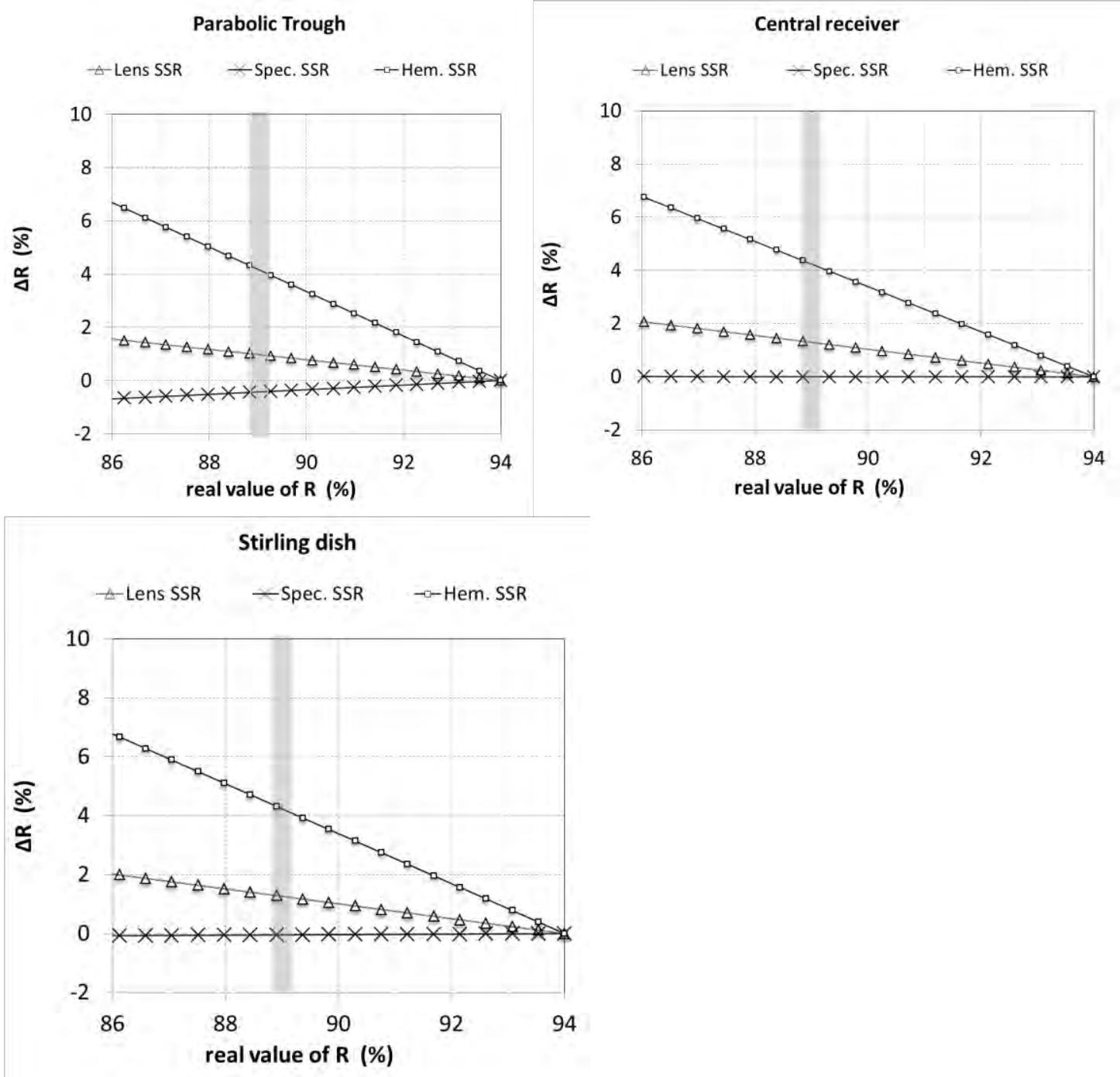

Figure 12. Error $\Delta R$ versus specular reflection coefficient for the different types of reflectometers and collectors, using a Mie scattering model for the diffuse reflection.

Therefore, the hemispherical SSR provides almost no information about the soiling rate of the solar field mirrors, although for clean conditions it is highly tolerant to changes in geometry (as the measurement does not depend on the direction of the incident ray).

On the other hand, the specular SSR gives a good estimation of the effective specular reflection coefficient, accurately discarding diffuse light, but this comes at the cost of a very low tolerance to changes in geometry, making it impossible to use the same SSR for different mirrors or even regions of the same curved mirror (unless movable parts are present, which is undesirable for fast and simple measurements as those required in solar field surveying).

Our design for a SSR achieves a compromise between these two models, providing tolerance enough to measure mirrors of various curvatures and thicknesses at the expense of a relative overestimation of the reflection coefficient. Even in the worst of the situations, this overestimation is under $1.35 \%$ in operation conditions, small enough to allow the scheduling of an adequate cleaning routine for a solar plant (Crawford et al., 2012). In a large survey consisting in a lot of repetitive measurements, the possibility of a measurement error due to handling and adjustments can be more relevant than the error due to diffuse light. 
These predictions fit with the measurements shown also in Crawford et al., 2012, where the three types of reflectometer are compared. In this study the specular SSR (Devices \&Services $15 R$ ) and an SSR designed as described in this paper (Condor from Abengoa) provide similar results for $R$, whereas the measurements obtained with a hemispherical SSR (Surface Optics Corporation 410 Solar) deviate strongly when compared to the other two.

The overestimation of the diffuse reflection could be further reduced with changes in the acceptance angle, for example with a reduction of the lens diameter, if lower uncertainty were required. This, however, would also reduce the tolerance to the alignment of the optical system, so it is important to define a careful balance.

The commercial reflectometer Condor serves as a field test of the advantages described on this work. The compromise between tolerance to changes in the optical path (due to changes in the geometry of the mirrors, wrong positioning or dirt) and tolerance to diffuse reflection, along with the absence of mobile parts to be adjusted, has made it suitable for the fast and reliable characterization of the solar field of different types of commercial STE plants distributed worldwide.

\section{Conclusions}

A new field Solar Spectrum Reflectometer for solar-weighted specular reflectance characterization of planar, spherical or parabolic mirrors has been presented. It is based on the use of collecting lenses in the detection system, which provides the necessary tolerance to changes in the geometrical dimensions of the mirrors, as an improvement over the existing SSR design strategies. This device is able to characterize mirrors up to $5 \mathrm{~mm}$ in thickness and curvature radius down to $300 \mathrm{~mm}$ without losing accuracy and without needing any mechanical adjustments. Therefore, its design is adequate to characterize all the different kinds of mirrors used in solar thermal technology, either in Stirling, central tower or parabolic through solar plants.

The influence of diffuse reflection and its impact on the measured reflectance has been calculated for different types of collectors, using both Lambertian and Mie diffuse reflection models. These results compare favorably to the performance of hemispherical and specular SSRs.

\section{Acknowledgements}

The authors want to thank the Spanish Ministerio de Economía y Competitividad for the funding through grant IPT-2011-1425-920000.

\section{References}

Crawford J.S., Stewart, J., Ganza, S., Asensio, J., 2012. A comparison of three portable reflectometers for use in operation and maintenance of CSP plants. SolarPACES conference proceedings, Marrakech, Morocco.

Fend T., Hoffschmidt, B., Jorgensen, G., Küster, H., Krüger, D., Pitz-Paal, R., Rietbrock, P., Riffelmann, K.J., 2003. Comparative assessment of solar concentrator materials. Solar Energy 74, 149-155.

Heller, P., 2013. Solar Paces Guideline: Parameters and Method to Evaluate the Solar Reflectance Properties of Reflector Materials for Concentrating Solar Power Technology, version2.5, June2013. Online: 〈http://www.solarpaces.org/tasks/task-iii-solar-technology-andadvanced-applications $\rangle$ 
Levinson R., Akbari, H., Berdahl, P., 2010. Measuring Solar Reflectance-part II: Review of practical methods. Solar Energy 84, 1745-1759.

Meyen, S., Lüpfert, E., Pernpeintner, J., Fend, T., 2009. Optical Characterization of Reflector Material for Concentrating Solar Power Technology. SolarPACES conference proceedings, Berlin, Germany.

Meyen S., Fernández-García, A., Kennedy, C., Lüpfert, E., 2010. Standardization of Solar Mirror Reflectance Measurements - Round Robin Test. SolarPACES conference proceedings, Perpignan, France.

Montecchi, M., 2013. Approximated method for modelling hemispherical reflectance and evaluating near-specular reflectance of CSP mirrors. Solar Energy 92, 280-287

Pettit, R.B., 1977. Characterization of the reflected beam profile of solar mirror materials. Solar Energy 19, 743-741.

Pettit, R.B., Freese, J.M., 1980. Wavelength dependent scattering caused by dust accumulation on solar mirrors. Solar Energy Materials 3 (1-2), 1-20.

Pettit, R.B., 1982. Characterizing Solar Mirror Materials Using Portable Reflectometer. Sandia Report, Albuquerque, SAND82-1714.

Polato, P., Masetti, E., 1988. Reflectance measurements of second-surface mirrors using commercial spectrophotometer accessories. Solar Energy 41 (5) 443-452.

Roos, A., 1993. Use of an integrating sphere in solar energy research. Solar Energy Materials and Solar Cells 30 (1), 77-94.

Sutter, F., Meyen, S., Fernández-García, A., Heller, P., 2016. Spectral characterization of specular reflectance of solar mirrors. Solar EnergyMaterials\&SolarCells 145, 248-254. doi:10.1016/j.solmat.2015.10.030

Zhang H., Baeyens J., Degrève J., Cacères G., 2013. Concentrated solar power plants: review and design methodology, Renewable and Sustainable Energy Reviews 22, 466-481. 